[Supporting Information]

\title{
Machine Learning Prediction on Properties of Nanoporous Materials utilizing Pore Geometry Barcodes
}

\author{
Xiangyu Zhang, Jing Cui, Kexin Zhang, Jiasheng Wu, and Yongjin Lee*
}

School of Physical Science and Technology, ShanghaiTech University, Shanghai 201210, China
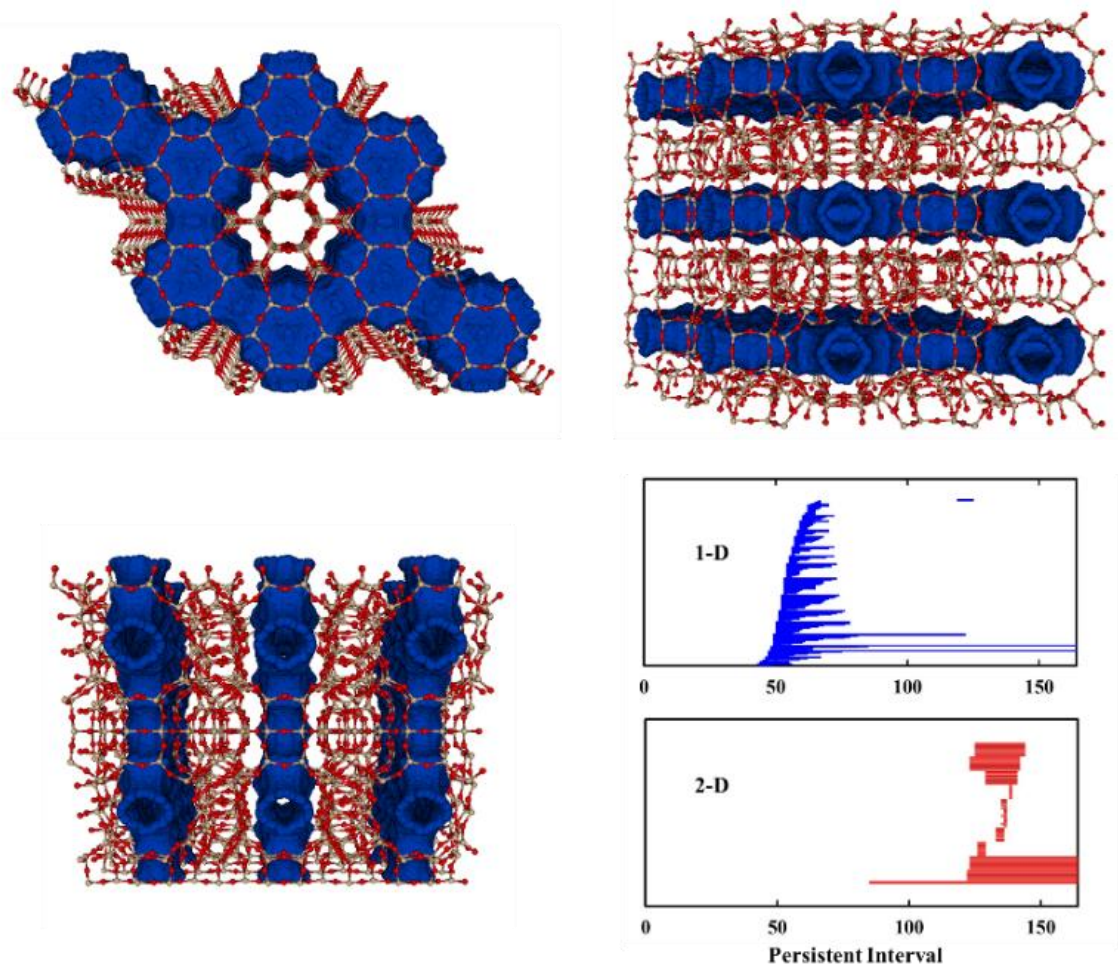

Figure S1. Example of pore geometry barcodes for zeolite SSF. In top and bottom left pictures, zeolite SSF (ball and stick model) and pore structure (blue colored shape) are shown from different orientations. At the bottom right, 1- and 2- dimensional barcodes are shown. In each barcode plot, one persistent interval corresponds to $0.025 \AA$. 
(a)
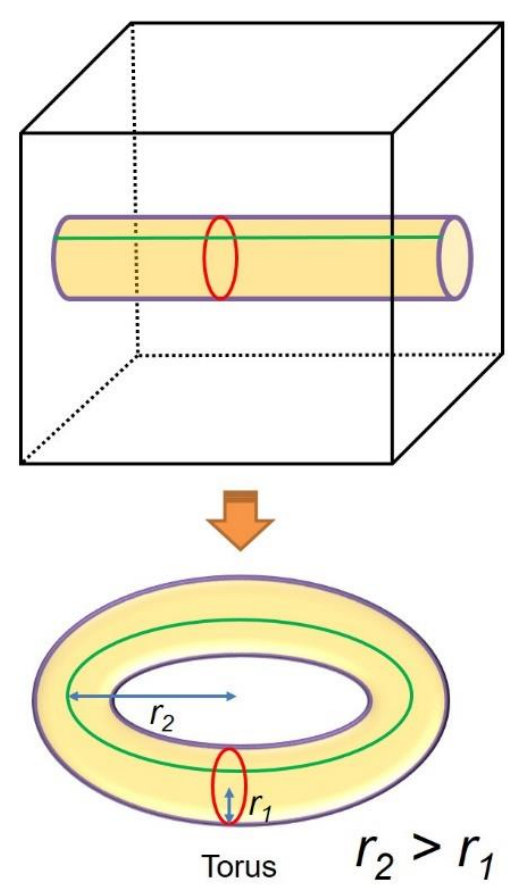

$H_{0}$

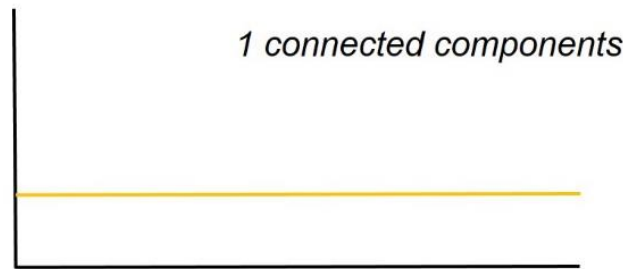

$H_{1}$

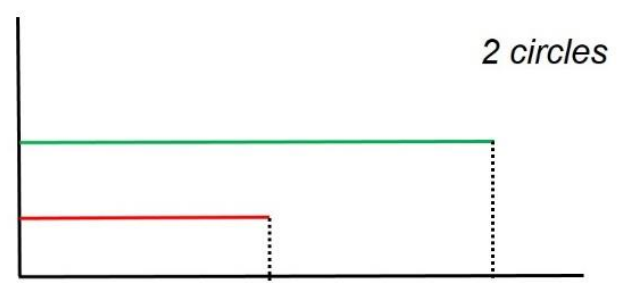

$\mathrm{H}_{2}$

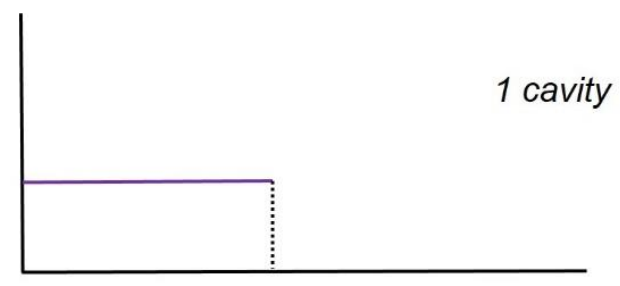

(b)

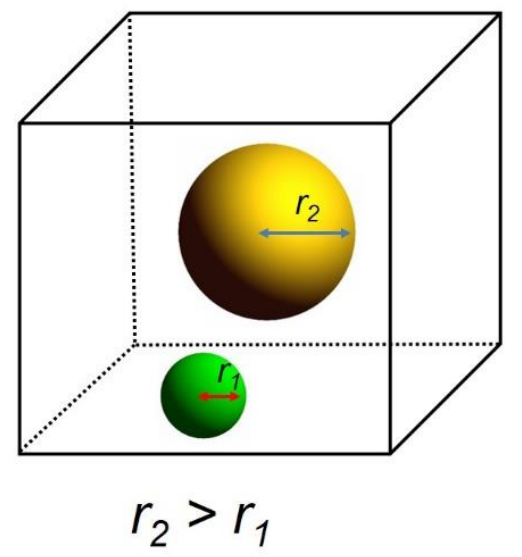

$H_{0}$

2 connected components

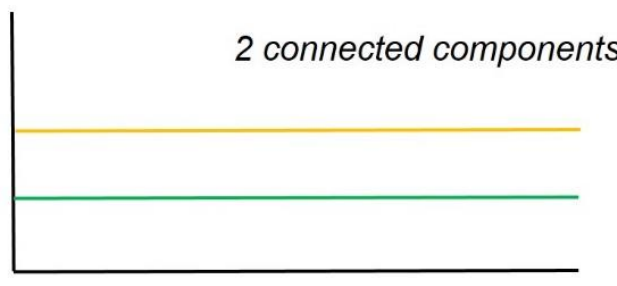

$H_{2}$

2 pockets

Figure S2. An example of persistence barcodes obtained from (a) a pore channel and (b) twopore pockets with periodic boundary conditions. Each bar has color of the corresponding topological feature. 

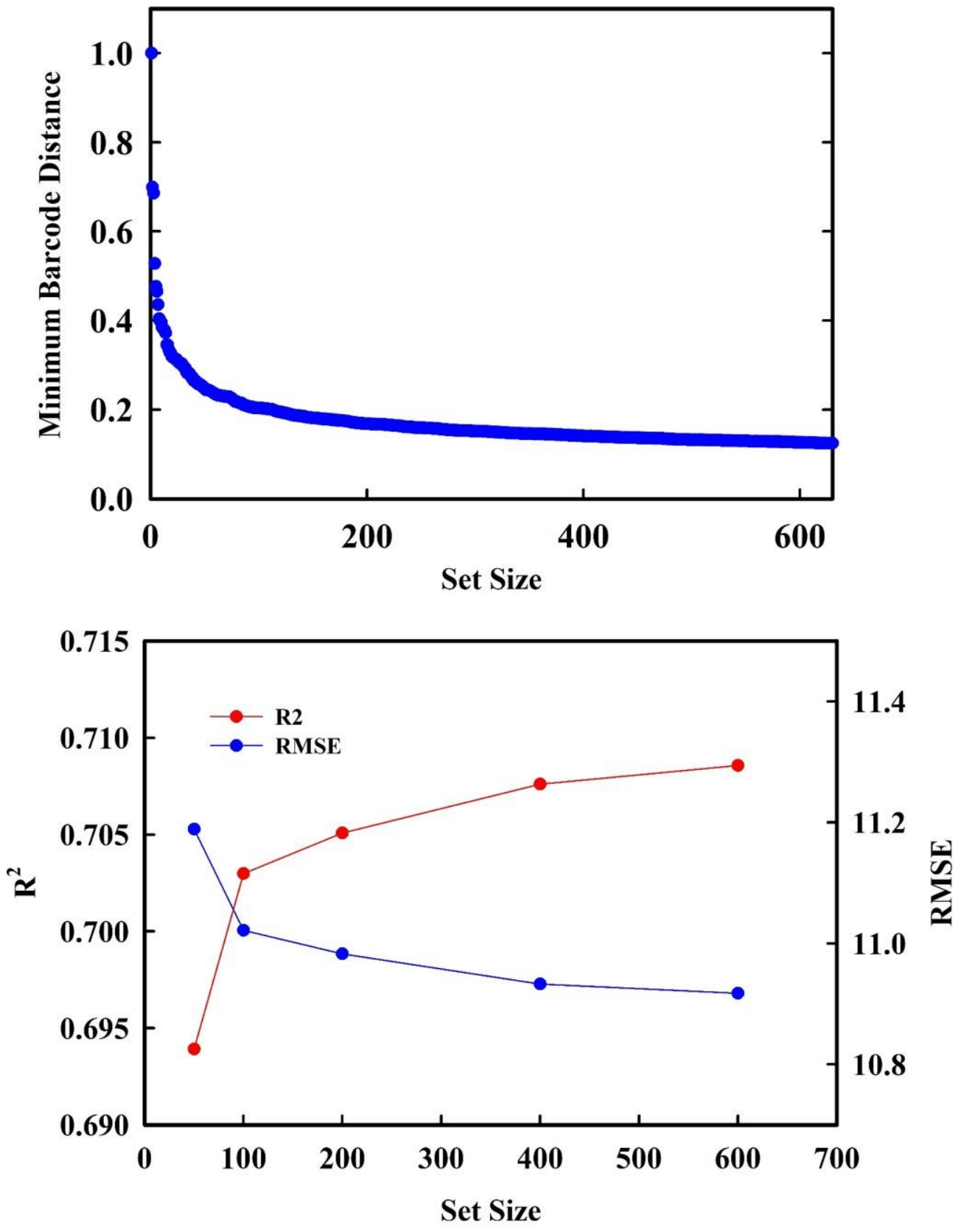

Figure S3. The variation of minimum barcode distance (top) and prediction accuracy (bottom) as a function of the most diverse set size. 


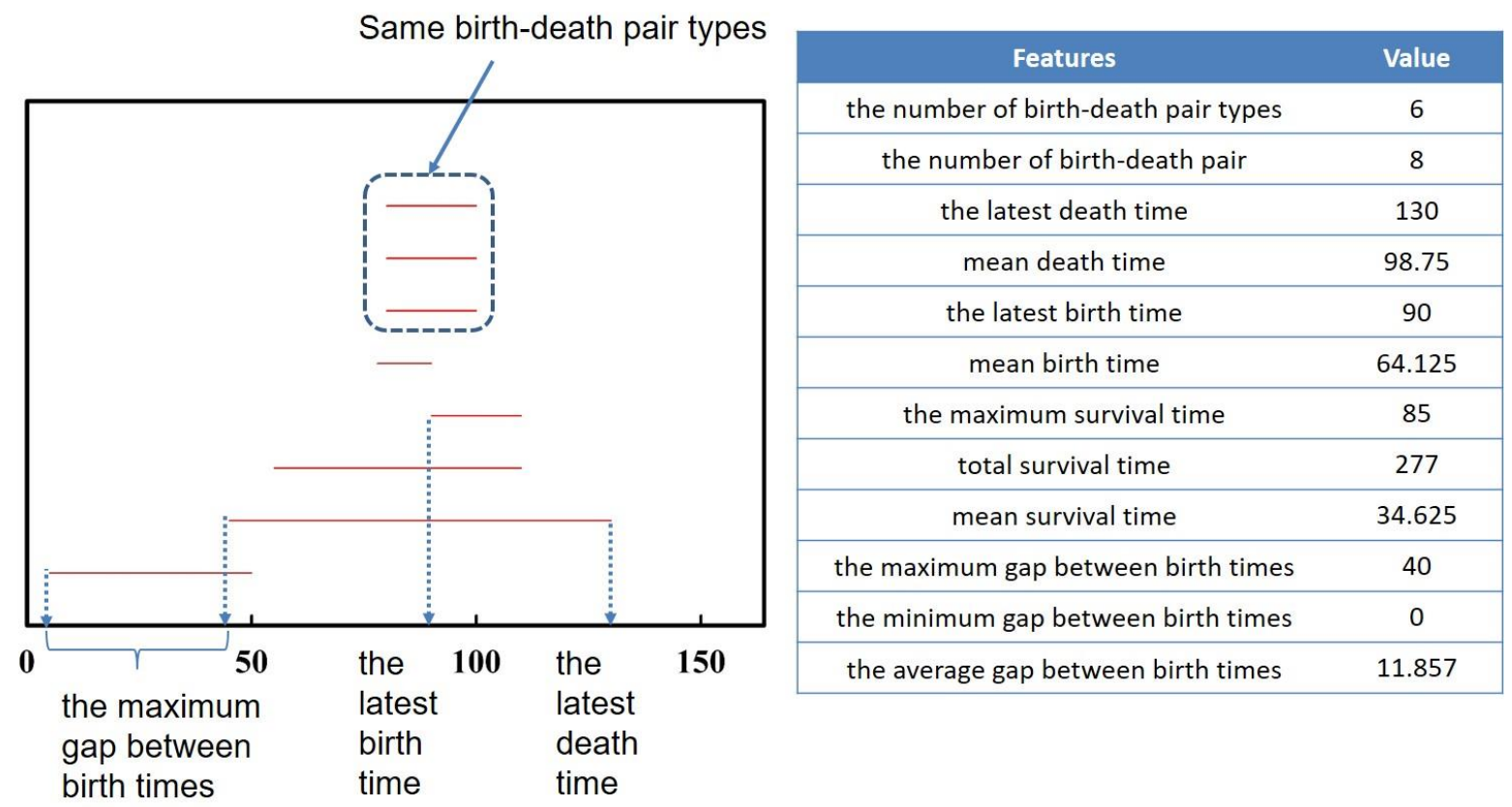

Figure S4. An example of a barcode and its features. The descriptions about features are as follows. The number of birth-death pairs equals the number of bars. In counting the number of birth-death pair types, bars having the same birth and death times are regarded as one pair type. The latest birth or death time is the persistent interval value at staring or closing point of a bar, respectively. Mean birth or death time is defined as \{sum of birth or death time\}/\{the number of birth-death pair $\}$, respectively. Maximum survival time is the largest lifetime among all bars. Total survival time is summation of lifetimes of all bars, and mean survival time is estimated by dividing total survival time with the number of birth-death pair. The maximum or minimum gap between birth times is magnitude of largest or smallest interval range where no bar appears, respectively. The average gap between birth times is obtained as sum of all birth time gaps $\} /\{$ total number of gaps\}, where total number of gaps is the number of birth-death pair $1\}$. 

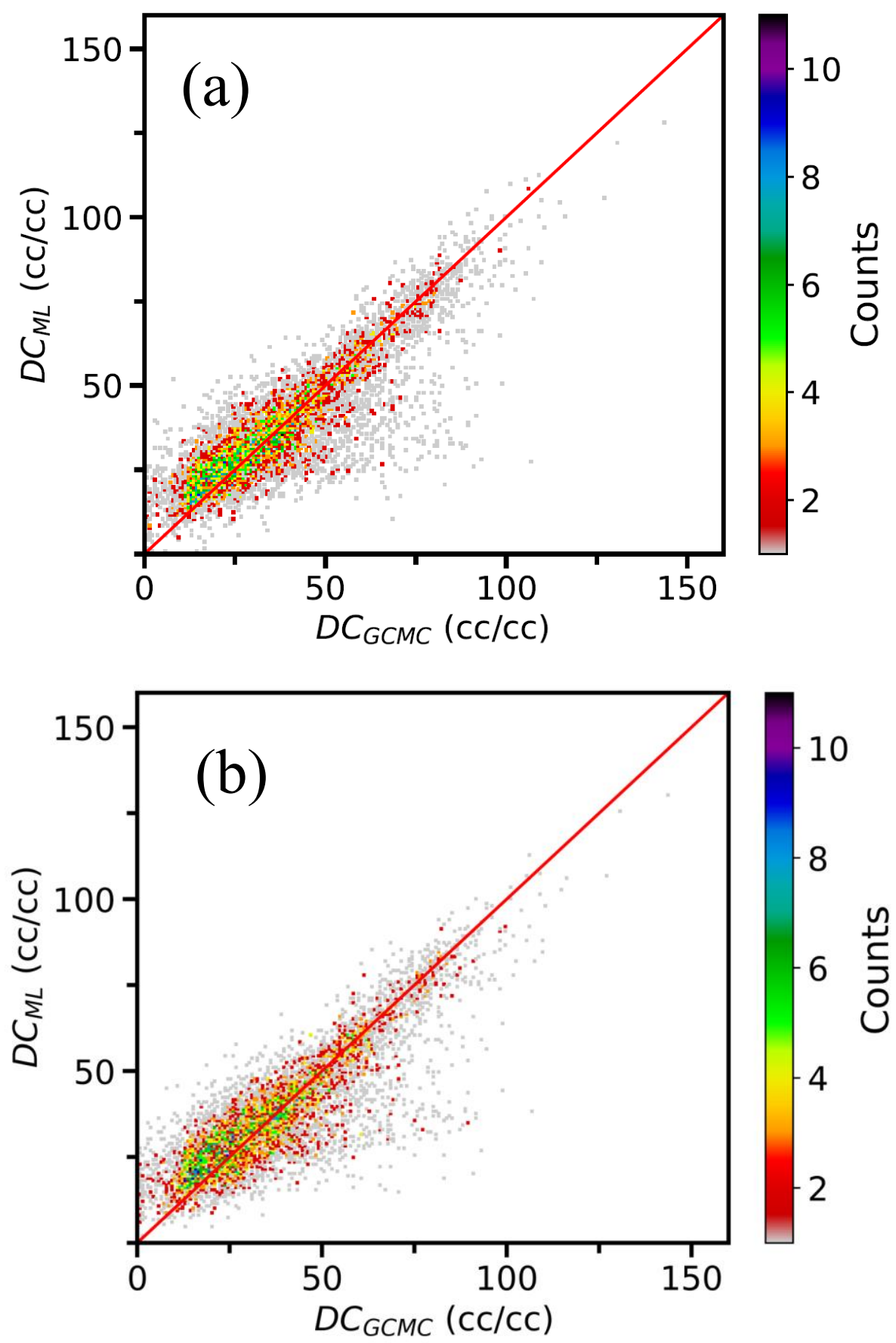

Figure S5. Parity plots of deliverable capacity $(D C)$ for training set structures using kernel ridge regression model with (a) D_mif and (b) D_mds. $D C_{\mathrm{GCMC}}$ is $D C$ from grand canonical Monte Carlo simulation and $D C_{\mathrm{ML}}$ is from machine learning. The color bar indicates a number of structures. 

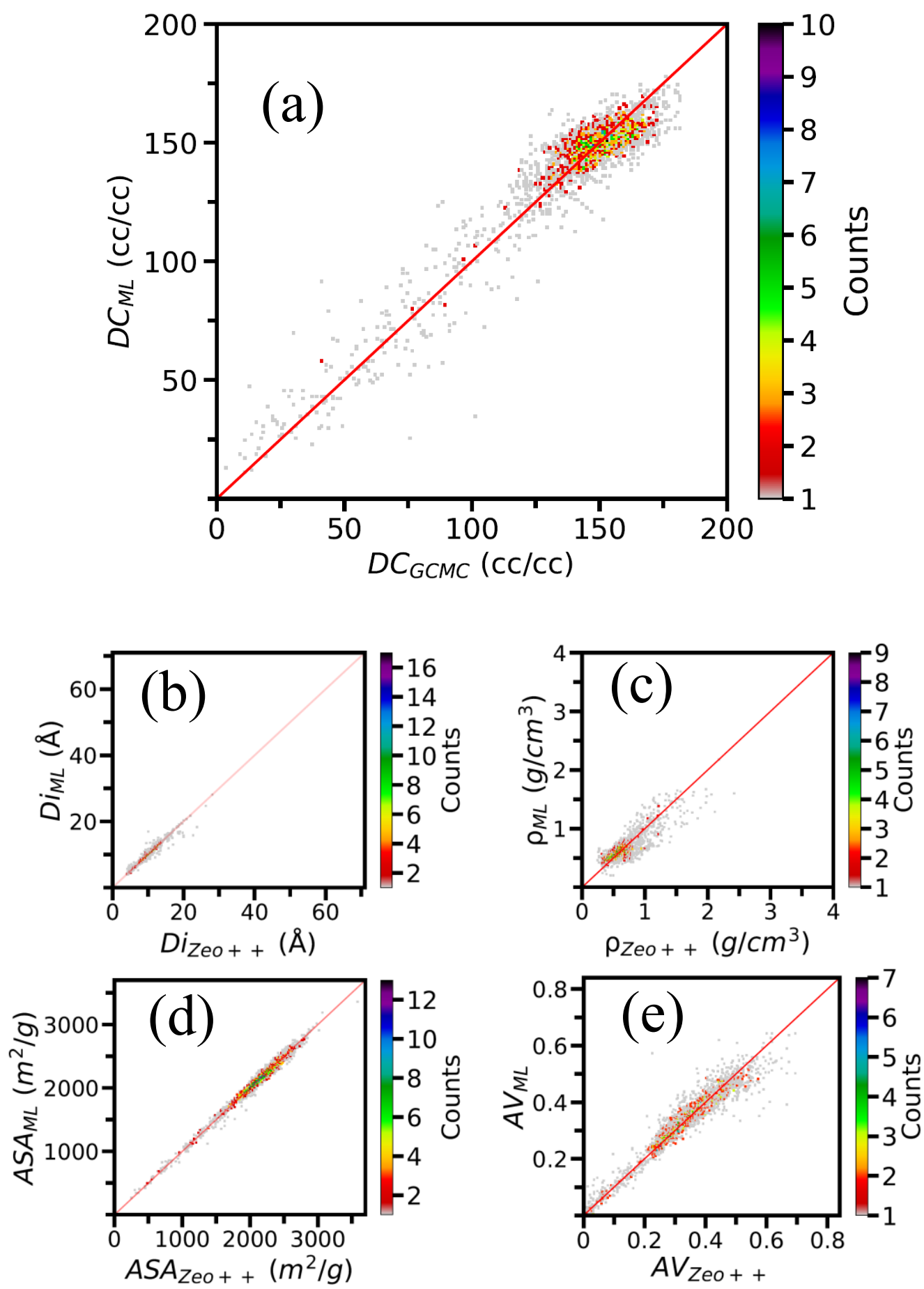

Figure S6. Parity plots on properties of metal-organic frameworks in the training set using kernel ridge regression model with D_mif; (a) Deliverable capacity $(D C)$ (b) $\mathrm{D}_{\mathrm{i}}$, (c) $\rho$, (d) ASA, (e) AV. In each graph, the $\mathrm{x}$-axis represents properties calculated by grand canonical Monte Carlo (for $D C$ ) or Zeo++ (for the other properties). The y-axis shows properties by machine learning. 
Table S1. List of top 600 most diverse set of zeolites. '\#' indicates an order of being added in the most diverse set.

\begin{tabular}{|c|c|c|c|c|c|c|c|}
\hline \# & Name & \# & Name & \# & Name & \# & Name \\
\hline 1 & PCOD8210717 & 151 & PCOD8294015 & 301 & PCOD8136747 & 451 & PCOD8319069 \\
\hline 2 & PCOD8036699 & 152 & PCOD8065733 & 302 & PCOD8123883 & 452 & PCOD8038830 \\
\hline 3 & PCOD8330909 & 153 & PCOD8329161 & 303 & PCOD8326129 & 453 & PCOD8200798 \\
\hline 4 & PCOD8265081 & 154 & PCOD8200990 & 304 & PCOD8325731 & 454 & PCOD8001316 \\
\hline 5 & PCOD8065780 & 155 & PCOD8060458 & 305 & PCOD8124960 & 455 & PCOD8200897 \\
\hline 6 & PCOD8331105 & 156 & PCOD8194806 & 306 & RWY & 456 & PCOD8095027 \\
\hline 7 & PCOD8319194 & 157 & PCOD8310673 & 307 & PCOD8067936 & 457 & PCOD8320217 \\
\hline 8 & PCOD8075669 & 158 & PCOD8322839 & 308 & PCOD8018248 & 458 & PCOD8085019 \\
\hline 9 & TSC & 159 & PCOD8067623 & 309 & PCOD8320019 & 459 & PCOD8328654 \\
\hline 10 & PCOD8239064 & 160 & PCOD8292631 & 310 & PCOD8038624 & 460 & PCOD8200887 \\
\hline 11 & PCOD8328352 & 161 & PCOD8323414 & 311 & PCOD8320011 & 461 & PCOD8167032 \\
\hline 12 & PCOD8118501 & 162 & PCOD8250356 & 312 & PCOD8325389 & 462 & PCOD8279061 \\
\hline 13 & PCOD8160769 & 163 & PCOD8328247 & 313 & PCOD8118653 & 463 & PCOD8313571 \\
\hline 14 & PCOD8059368 & 164 & PCOD8275575 & 314 & PCOD8328489 & 464 & PCOD8309154 \\
\hline 15 & PCOD8118629 & 165 & PCOD8080023 & 315 & PCOD8162096 & 465 & PCOD8331035 \\
\hline 16 & PCOD8326820 & 166 & PCOD8137957 & 316 & PCOD8318331 & 466 & PCOD8275111 \\
\hline 17 & PCOD8328649 & 167 & PCOD8318566 & 317 & PCOD8214861 & 467 & PCOD8038621 \\
\hline 18 & PCOD8330288 & 168 & PCOD8162399 & 318 & PCOD8313064 & 468 & PCOD8059584 \\
\hline 19 & PCOD8009302 & 169 & PCOD8200971 & 319 & PCOD8078626 & 469 & PCOD8136416 \\
\hline 20 & PCOD8115519 & 170 & PCOD8067988 & 320 & PCOD8070586 & 470 & PCOD8312991 \\
\hline 21 & PCOD8067951 & 171 & PCOD8075785 & 321 & PCOD8066560 & 471 & PCOD8275509 \\
\hline 22 & PCOD8038664 & 172 & PCOD8059734 & 322 & PCOD8095104 & 472 & PCOD 8323840 \\
\hline 23 & PCOD8067898 & 173 & PCOD8001055 & 323 & PCOD8331112 & 473 & PCOD8014051 \\
\hline 24 & PCOD8222251 & 174 & PCOD8065386 & 324 & PCOD8324915 & 474 & PCOD8058099 \\
\hline 25 & PCOD8322926 & 175 & PCOD8312182 & 325 & PCOD8057878 & 475 & PCOD8328264 \\
\hline 26 & PCOD8066909 & 176 & PCOD8214697 & 326 & PCOD8003815 & 476 & PCOD8310028 \\
\hline 27 & PCOD8322938 & 177 & PCOD8202219 & 327 & PCOD8065431 & 477 & PCOD8328398 \\
\hline 28 & PCOD8283909 & 178 & PCOD8323362 & 328 & PCOD8315726 & 478 & PCOD8318333 \\
\hline 29 & PCOD8078042 & 179 & PCOD8274868 & 329 & PCOD8284133 & 479 & PCOD8201062 \\
\hline 30 & PCOD8117134 & 180 & PCOD8328375 & 330 & PCOD8105342 & 480 & PCOD8195860 \\
\hline
\end{tabular}




\begin{tabular}{|c|c|c|c|c|c|c|c|}
\hline 31 & AFY & 181 & PCOD8314987 & 331 & PCOD8119183 & 481 & PCOD8052688 \\
\hline 32 & PCOD8117002 & 182 & PCOD8200774 & 332 & PCOD8331110 & 482 & PCOD8160758 \\
\hline 33 & PCOD8063915 & 183 & PCOD8085120 & 333 & PCOD8322943 & 483 & PCOD8069018 \\
\hline 34 & PCOD8328572 & 184 & PCOD8065391 & 334 & PCOD8067418 & 484 & PCOD8079820 \\
\hline 35 & PCOD8317676 & 185 & PCOD8284020 & 335 & PCOD8059475 & 485 & PCOD8279493 \\
\hline 36 & PCOD8041656 & 186 & PCOD8323377 & 336 & PCOD8232225 & 486 & PCOD8065387 \\
\hline 37 & PCOD8124726 & 187 & PCOD8321706 & 337 & PCOD8125310 & 487 & PCOD8056908 \\
\hline 38 & PCOD8085302 & 188 & PCOD8118547 & 338 & PCOD8308137 & 488 & PCOD8129695 \\
\hline 39 & PCOD8202179 & 189 & PCOD8292616 & 339 & PCOD8303367 & 489 & PCOD 8274883 \\
\hline 40 & PCOD8202550 & 190 & PCOD8054184 & 340 & PCOD8057871 & 490 & PCOD8313115 \\
\hline 41 & PCOD8309175 & 191 & PCOD8056880 & 341 & PCOD8067907 & 491 & PCOD8059621 \\
\hline 42 & PCOD8088436 & 192 & PCOD8285679 & 342 & PCOD8321679 & 492 & PCOD8312183 \\
\hline 43 & PCOD8316520 & 193 & PCOD8274910 & 343 & PCOD8124042 & 493 & PCOD8002033 \\
\hline 44 & PCOD8312990 & 194 & PCOD8168034 & 344 & PCOD8004498 & 494 & PCOD 8310021 \\
\hline 45 & PCOD8277922 & 195 & PCOD8115833 & 345 & PCOD8207498 & 495 & PCOD8067892 \\
\hline 46 & PCOD8308355 & 196 & PCOD8070472 & 346 & PCOD8065945 & 496 & PCOD 8286928 \\
\hline 47 & PCOD8331109 & 197 & PCOD8066397 & 347 & PCOD8041658 & 497 & PCOD8193692 \\
\hline 48 & PCOD8079751 & 198 & PCOD8060230 & 348 & PCOD8331106 & 498 & PCOD8059640 \\
\hline 49 & PCOD8328643 & 199 & PCOD8085621 & 349 & PCOD8200977 & 499 & PCOD 8218580 \\
\hline 50 & PCOD8281580 & 200 & PCOD8201179 & 350 & PCOD8167200 & 500 & PCOD8117977 \\
\hline 51 & PCOD8028876 & 201 & PCOD8312134 & 351 & PCOD8116842 & 501 & PCOD8277946 \\
\hline 52 & PCOD8200996 & 202 & PCOD8058229 & 352 & PCOD8201160 & 502 & PCOD8275022 \\
\hline 53 & PCOD8331107 & 203 & PCOD8329048 & 353 & PCOD8197038 & 503 & PCOD8197570 \\
\hline 54 & PCOD8315657 & 204 & PCOD8272906 & 354 & PCOD8325391 & 504 & PCOD8316389 \\
\hline 55 & PCOD8065956 & 205 & PCOD8068653 & 355 & PCOD8008656 & 505 & PCOD8284297 \\
\hline 56 & PCOD8141030 & 206 & PCOD8321600 & 356 & PCOD8329282 & 506 & PCOD8324714 \\
\hline 57 & PCOD8057562 & 207 & PCOD8105244 & 357 & PCOD8078093 & 507 & PCOD8260934 \\
\hline 58 & PCOD8331157 & 208 & PCOD8167786 & 358 & PCOD8292645 & 508 & PCOD8119785 \\
\hline 59 & PCOD8043492 & 209 & PCOD8289701 & 359 & PCOD8269798 & 509 & PCOD8246139 \\
\hline 60 & PCOD8329285 & 210 & PCOD8160766 & 360 & PCOD8067894 & 510 & PCOD8193458 \\
\hline 61 & PCOD8069233 & 211 & PCOD8210459 & 361 & PCOD8085257 & 511 & PCOD8328822 \\
\hline 62 & PCOD8003468 & 212 & PCOD8002601 & 362 & PCOD8059349 & 512 & PCOD8007263 \\
\hline 63 & PCOD8029622 & 213 & PCOD8209201 & 363 & PCOD8123228 & 513 & PCOD8130824 \\
\hline
\end{tabular}




\begin{tabular}{|c|c|c|c|c|c|c|c|}
\hline 64 & PCOD8316376 & 214 & PCOD8328263 & 364 & PCOD8317689 & 514 & PCOD8193729 \\
\hline 65 & PCOD8200782 & 215 & PCOD8131316 & 365 & PCOD8168320 & 515 & PCOD8059739 \\
\hline 66 & PCOD8204327 & 216 & PCOD8041689 & 366 & PCOD8095408 & 516 & PCOD8200915 \\
\hline 67 & PCOD8041734 & 217 & PCOD8278735 & 367 & PCOD8177194 & 517 & PCOD8319440 \\
\hline 68 & PCOD8200584 & 218 & PCOD8265066 & 368 & PCOD8200810 & 518 & PCOD8128924 \\
\hline 69 & PCOD8331103 & 219 & PCOD8279607 & 369 & PCOD8297090 & 519 & PCOD8196901 \\
\hline 70 & PCOD8238992 & 220 & PCOD8041761 & 370 & PCOD8322840 & 520 & PCOD8322951 \\
\hline 71 & PCOD8307992 & 221 & PCOD8331120 & 371 & PCOD8310033 & 521 & PCOD8070356 \\
\hline 72 & PCOD8068221 & 222 & PCOD8069115 & 372 & PCOD8067880 & 522 & PCOD8279511 \\
\hline 73 & PCOD8029580 & 223 & PCOD8265203 & 373 & PCOD8329576 & 523 & PCOD8059613 \\
\hline 74 & PCOD8319023 & 224 & PCOD8016769 & 374 & PCOD8095804 & 524 & PCOD8129532 \\
\hline 75 & PCOD8310684 & 225 & PCOD8059577 & 375 & PCOD8079021 & 525 & PCOD8329360 \\
\hline 76 & PCOD8267101 & 226 & PCOD8199650 & 376 & PCOD8056852 & 526 & PCOD8317715 \\
\hline 77 & PCOD8330836 & 227 & PCOD8062952 & 377 & PCOD8312316 & 527 & PCOD8063082 \\
\hline 78 & PCOD8066395 & 228 & PCOD8061391 & 378 & PCOD8065706 & 528 & PCOD8330866 \\
\hline 79 & PCOD8006394 & 229 & PCOD8319455 & 379 & PCOD8275003 & 529 & PCOD8122708 \\
\hline 80 & PCOD8306412 & 230 & PCOD8119353 & 380 & PCOD8200989 & 530 & PCOD8059456 \\
\hline 81 & PCOD8275239 & 231 & PCOD8274946 & 381 & PCOD8230728 & 531 & PCOD8304176 \\
\hline 82 & PCOD8115961 & 232 & PCOD8312508 & 382 & PCOD8155190 & 532 & PCOD8096201 \\
\hline 83 & PCOD8064806 & 233 & PCOD8163185 & 383 & PCOD8294105 & 533 & PCOD8016820 \\
\hline 84 & PCOD8200918 & 234 & PCOD8318326 & 384 & PCOD8117043 & 534 & PCOD8132981 \\
\hline 85 & PCOD8329307 & 235 & PCOD8331136 & 385 & PCOD8121312 & 535 & PCOD8068127 \\
\hline 86 & PCOD8320469 & 236 & PCOD8163826 & 386 & PCOD8060459 & 536 & PCOD 8323850 \\
\hline 87 & PCOD8307180 & 237 & PCOD8213838 & 387 & PCOD8195665 & $\mathbf{5 3 7}$ & PCOD8060094 \\
\hline 88 & PCOD8033743 & 238 & PCOD8197998 & 388 & PCOD8290384 & 538 & PCOD8274935 \\
\hline 89 & PCOD8118101 & 239 & PCOD8281655 & 389 & PCOD8319381 & 539 & PCOD8048954 \\
\hline 90 & PCOD8059552 & 240 & PCOD8267076 & 390 & PCOD8080710 & 540 & PCOD8265155 \\
\hline 91 & PCOD8330376 & 241 & PCOD8029979 & 391 & PCOD8261276 & 541 & PCOD8298485 \\
\hline 92 & PCOD8321916 & 242 & PCOD8269929 & 392 & PCOD8180519 & 542 & PCOD8214698 \\
\hline 93 & PCOD8303120 & 243 & PCOD8323346 & 393 & PCOD8059549 & 543 & PCOD8319479 \\
\hline 94 & PCOD8239851 & 244 & PCOD8200741 & 394 & PCOD8199249 & 544 & PCOD8115975 \\
\hline 95 & PCOD8298158 & 245 & PCOD8312528 & 395 & PCOD8085942 & 545 & PCOD8325726 \\
\hline 96 & PCOD8062912 & 246 & PCOD8061696 & 396 & PCOD8328656 & 546 & PCOD8205920 \\
\hline
\end{tabular}




\begin{tabular}{|c|c|c|c|c|c|c|c|}
\hline 97 & RHO & 247 & PCOD8319270 & 397 & PCOD8067532 & 547 & PCOD8327284 \\
\hline 98 & PCOD8024574 & 248 & PCOD8067900 & 398 & PCOD8285676 & 548 & PCOD8200869 \\
\hline 99 & PCOD8285595 & 249 & PCOD8124711 & 399 & PCOD8297291 & 549 & PCOD8274921 \\
\hline 100 & PCOD8077931 & 250 & PCOD8200768 & 400 & PCOD8006389 & 550 & PCOD8123888 \\
\hline 101 & PCOD8329367 & 251 & PCOD8078160 & 401 & PCOD8266023 & 551 & PCOD8056807 \\
\hline 102 & PCOD8129343 & 252 & PCOD8162093 & 402 & PCOD8067909 & 552 & PCOD8193805 \\
\hline 103 & PCOD8167968 & 253 & PCOD8059738 & 403 & PCOD8061490 & 553 & PCOD8320537 \\
\hline 104 & PCOD8328295 & 254 & PCOD8302649 & 404 & PCOD8292617 & 554 & PCOD8057508 \\
\hline 105 & PCOD8078542 & 255 & PCOD8313042 & 405 & PCOD8305122 & 555 & PCOD8261268 \\
\hline 106 & PCOD8308802 & 256 & PCOD8325399 & 406 & PCOD8067540 & 556 & EMT \\
\hline 107 & PCOD8133553 & 257 & PCOD8065797 & 407 & PCOD8323347 & 557 & PCOD8180522 \\
\hline 108 & PCOD8193822 & 258 & PCOD8058201 & 408 & PCOD8041047 & 558 & PCOD8313047 \\
\hline 109 & PCOD8294332 & 259 & PCOD8318158 & 409 & PCOD8048174 & 559 & PCOD8180636 \\
\hline 110 & PCOD8057084 & 260 & PCOD8306952 & 410 & PCOD8326513 & 560 & PCOD8266261 \\
\hline 111 & PCOD8069015 & 261 & PCOD8201035 & 411 & PCOD 8090380 & 561 & SBT \\
\hline 112 & PCOD8328564 & 262 & PCOD8067939 & 412 & PCOD8043835 & 562 & PCOD8307680 \\
\hline 113 & PCOD8219220 & 263 & PCOD8060040 & 413 & PCOD8060322 & 563 & PCOD8129511 \\
\hline 114 & PCOD8323835 & 264 & PCOD8214713 & 414 & PCOD8002219 & 564 & PCOD8274974 \\
\hline 115 & PCOD8085224 & 265 & PCOD8323493 & 415 & PCOD8222700 & 565 & PCOD8059347 \\
\hline 116 & PCOD8106483 & 266 & PCOD8200916 & 416 & PCOD8082172 & 566 & PCOD8289594 \\
\hline 117 & PCOD8275521 & 267 & PCOD8186126 & 417 & PCOD8326705 & 567 & PCOD8326523 \\
\hline 118 & PCOD8116094 & 268 & PCOD8201019 & 418 & PCOD8067938 & 568 & PCOD8331050 \\
\hline 119 & PCOD8022660 & 269 & PCOD8057288 & 419 & PCOD8302898 & 569 & PCOD8200744 \\
\hline 120 & PCOD8178955 & 270 & PCOD8196981 & 420 & PCOD8077570 & 570 & PCOD8181303 \\
\hline 121 & PCOD8077937 & 271 & PCOD8180523 & 421 & PCOD8278506 & 571 & PCOD8274976 \\
\hline 122 & PCOD8313085 & 272 & PCOD8095248 & 422 & PCOD8201022 & 572 & PCOD 8268160 \\
\hline 123 & PCOD8328387 & 273 & PCOD8077967 & 423 & PCOD8060016 & 573 & PCOD8052712 \\
\hline 124 & PCOD8200519 & 274 & PCOD8329295 & 424 & PCOD8131624 & 574 & PCOD8080042 \\
\hline 125 & PCOD8323358 & 275 & PCOD8194978 & 425 & PCOD8268336 & 575 & PCOD8323436 \\
\hline 126 & PCOD8325386 & 276 & PCOD8238972 & 426 & PCOD8061259 & 576 & PCOD8010548 \\
\hline 127 & PCOD8193441 & 277 & PCOD8095788 & 427 & PCOD8118600 & 577 & PCOD 8057350 \\
\hline 128 & PCOD8123215 & 278 & PCOD8067914 & 428 & PCOD8043892 & 578 & PCOD8265208 \\
\hline 129 & PCOD8041037 & 279 & PCOD8162156 & 429 & PCOD8060402 & 579 & PCOD 8024870 \\
\hline
\end{tabular}




\begin{tabular}{|c|c|c|c|c|c|c|c|}
\hline 130 & PAU & 280 & PCOD8330930 & 430 & PCOD8268414 & 580 & PCOD8059345 \\
\hline 131 & PCOD8302672 & 281 & PCOD8323495 & 431 & PCOD8200950 & 581 & PCOD8160312 \\
\hline 132 & PCOD8205959 & 282 & PCOD8067904 & 432 & PCOD8312287 & 582 & AET \\
\hline 133 & PCOD8320563 & 283 & PCOD8166306 & 433 & PCOD8078159 & 583 & PCOD8329252 \\
\hline 134 & PCOD8060371 & 284 & PCOD8311724 & 434 & PCOD8328340 & 584 & PCOD8285688 \\
\hline 135 & PCOD8285677 & 285 & PCOD8309993 & 435 & PCOD8323388 & 585 & PCOD8200865 \\
\hline 136 & PCOD8214699 & 286 & PCOD8331160 & 436 & PCOD8313111 & 586 & PCOD8024126 \\
\hline 137 & PCOD8308298 & 287 & PCOD8200776 & 437 & PCOD8202262 & 587 & PCOD8095607 \\
\hline 138 & PCOD8267734 & 288 & PCOD8180580 & 438 & PCOD8205938 & 588 & PCOD8166595 \\
\hline 139 & PCOD8275709 & 289 & PCOD8200112 & 439 & PCOD8267023 & 589 & PCOD8283780 \\
\hline 140 & PCOD8327297 & 290 & PCOD8118945 & 440 & PCOD8319696 & 590 & PCOD8200817 \\
\hline 141 & PCOD8298846 & 291 & PCOD8082864 & 441 & PCOD8326827 & 591 & PCOD8200697 \\
\hline 142 & PCOD8078047 & 292 & PCOD8114120 & 442 & PCOD8056843 & 592 & PCOD8214714 \\
\hline 143 & PCOD8197324 & 293 & PCOD8177248 & 443 & PCOD8039026 & 593 & PCOD8321667 \\
\hline 144 & PCOD8224519 & 294 & PCOD8324006 & 444 & PCOD8321865 & 594 & PCOD8238971 \\
\hline 145 & PCOD8331163 & 295 & PCOD8279597 & 445 & PCOD8180890 & 595 & PCOD8271503 \\
\hline 146 & PCOD8322650 & 296 & PCOD8118506 & 446 & PCOD8328508 & 596 & PCOD8227633 \\
\hline 147 & PCOD8324936 & 297 & PCOD8319666 & 447 & PCOD8009223 & 597 & PCOD8292621 \\
\hline 148 & PCOD8313851 & 298 & PCOD8306855 & 448 & PCOD8134015 & 598 & PCOD8326521 \\
\hline 149 & PCOD8329857 & 299 & PCOD8309153 & 449 & PCOD8312225 & 599 & PCOD8243223 \\
\hline 150 & PCOD8306443 & 300 & PCOD8326706 & 450 & PCOD8331020 & 600 & PCOD8307101 \\
\hline
\end{tabular}

